\title{
3D multiscale segmentation and morphological analysis of x-ray microtomography from cold-sprayed coatings
}

\author{
L. GILliberT*, C. PEYREGA*, D. JEULIN*, V. GUIPONT $\dagger$ \\ $\&$ M. JEANDIN† \\ *Centre de Morphologie Mathématique, Mathématiques et Sytèmes, Mines ParisTech, France \\ †Centre des Matériaux, Mines ParisTech, CNRS UMR, France
}

Key words. Cold-sprayed coatings, constrained segmentation, multiscale 3D segmentation, stochastic watershed.

\begin{abstract}
Summary
X-ray microtomography from cold-sprayed coatings brings a new insight on this deposition process. A noisetolerant segmentation algorithm is introduced, based on the combination of two segmentations: a deterministic multiscale segmentation and a stochastic segmentation. The stochastic approach uses random Poisson lines as markers. Results on a X-ray microtomographic image of aluminium particles are presented and validated.
\end{abstract}

\section{Introduction}

The cold gas dynamic spray method or cold spray process is one of the more recent and innovative processes that has been introduced in the field of thermal spraying to achieve thick metallic layers. To characterize the microstructure resulting from the plastic deformation of the sprayed metallic particles by this process, X-ray microtomographic images of such materials were made on the synchrotron at the ESRF (Guipont et al., 2010; Rolland et al., 2010).

Two algorithms to segment the particles on these 3D images were implemented from methods using mathematical morphology such as the watershed transform (Beucher \& Lantuéjoul, 1979; Beucher, 1994). The watershed transformation used is an unbiased implementation based on hierarchical queues described in (Beucher, 2004).

The first algorithm is multiscale and consists of processing successive deterministic constrained watershed transforms to extract all the boundaries of the particles. The second one is based on the stochastic watershed segmentation which was first introduced by Angulo and Jeulin in (Angulo \& Jeulin,

Correspondence to: L. Gillibert, Centre de Morphologie Mathématique Mathématiques et systèmes, Mines ParisTech, 35, rue saint Honoré Fontaineloeav, France. Tel: +33164694792; Fax: +33164694707; e-mail: luc.gillibert@cmm.ensmp.fr
2007). The idea is to use a large number of realizations of random markers to build a probability density function (PDF) of contours, starting from a standard watershed algorithm producing oversegmentations. Here, random lines are used as markers. The material is anisotropic and therefore the lines will have a specific orientation.

Finally, the combination of the two algorithms is used. Together, they provide a relevant segmentation, multiscale and anisotropic.

\section{The cold spray coating method}

In this study, the metallic particles are spherical and composed of pure aluminium having an average diameter equal to $40 \mu \mathrm{m}$ as shown in Figure 1. The cold spray facility used at Mines ParisTech (K3000, CGT, Ampfing, Germany) is represented in Figure 2. Details for sample preparation are given in (Guipont et al., 2010). The particle size distribution of the powder was estimated by laser granulometry with a Mastersizer device (Malvern Instruments Ltd, England).

In conventional thermal spray processes, the pulverulent material is held in a liquid state when sprayed. The cold gas dynamic spray process only rests on the propulsion of particles at a high-velocity (300-200 $\mathrm{m} \mathrm{s}^{-1}$ typically) without fusion before impact. Therefore, the cold gas dynamic spray method could be defined as a solid-state process to prepare thick metallic layers from powder accelerated by a supersonic jet of compressed gas, with a De Laval nozzle. Cold sprayed particles are plastically deformed and no oxidation occurs during spraying. It results with a very original microstructure of deposited metals from a simultaneous deformation-agglomeration of particles keeping the purity and composition of the starting powder. It makes this processing route unique, simple and original to achieve dense or slightly porous pure metallic coatings. 


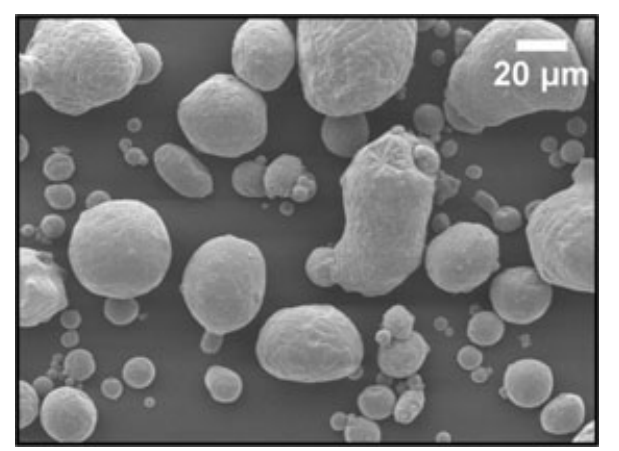

Fig. 1. SEM image of pure aluminium particles ; spherical particles (average diameter: $40 \mu \mathrm{m}$ ).

\section{Multiscale segmentation}

\section{Image acquisition}

For this study, thick aluminium coatings have been achieved by cold gas dynamic spraying at the Material Research Center (Mines ParisTech). Moreover, an in-depth impregnation by an appropriate etching solution composed of gallium has been achieved to reveal cold spray aluminium particles boundaries for further image segmentations. To characterize the microstructure of such a coating, 3D X-Ray microtomography images were obtained at the European Synchrotron Radiation Facility (ESRF). The resulting images were then processed to segment the deformed particles. The Figure 3(A) shows a 3D X-ray microtomographic reconstruction of a cold sprayed coating sample. The region of interest in Figure 3(B) is the sub-sample which was used to develop the segmentation algorithms presented in this paper. The method is validated on a second region of interest.

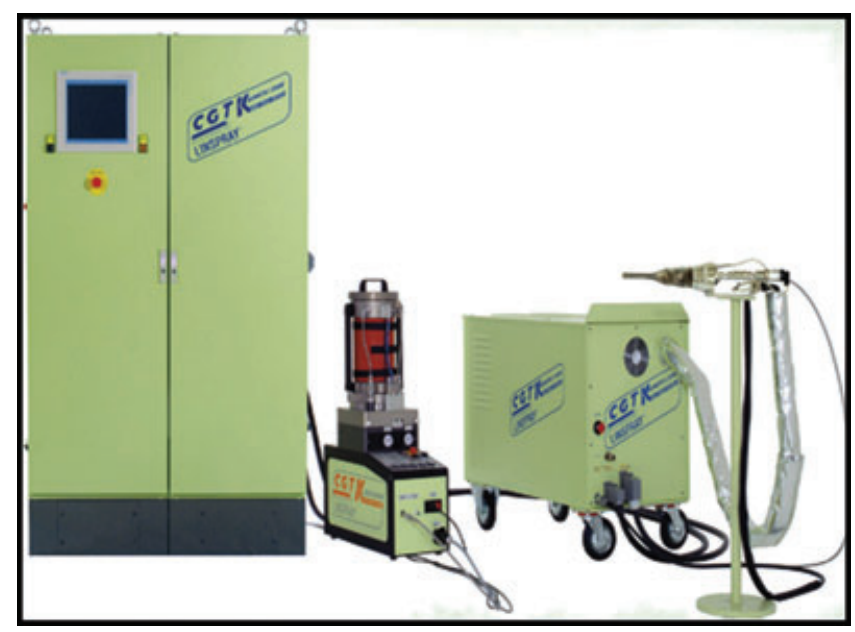

Fig. 2. Cold spray facility at Mines ParisTech (K3000, CGT, Ampfing, Germany).

\section{Multiscale image segmentation}

The first method handled to segment the particles is based on successive 3D watershed transforms with markers determined in advance by thresholding the original image. These markers are eroded at each iteration to correctly segment small and large particles in a multiscale approach.

The final segmentation is the union of the different watershed transforms (Guipont et al., 2010; Rolland et al., 2010). The algorithm 1 of the multiscale method is described below.

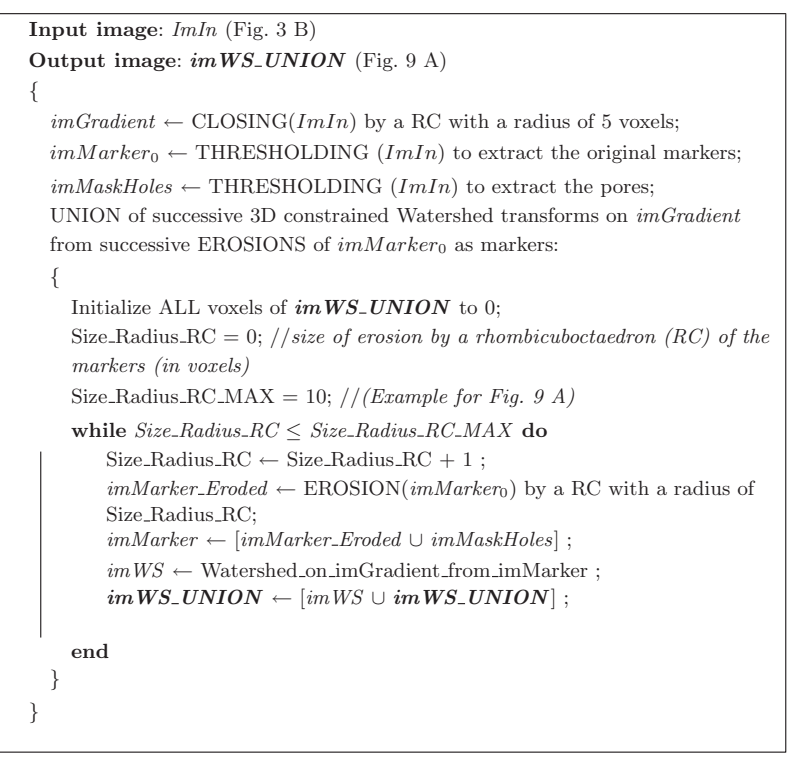

Algorithm 1. Multiscale segmentation.

To flood a smoother image, the closed image (Fig. 4) of the original one is used as a topological function for the watershed construction (step 1). Moreover, the pores are extracted by a manual thresholding of the original image to properly segment their boundaries. That is why they are included into the markers used for the constrained flooding (imMarker $\leftarrow$ [imMarker_Eroded $\cup$ imMaskHoles] in step 4).

The Figure 5 highlights the different steps of the multiscale segmentation method. The successive constrained watershed transforms are processed on imGradient from the white phase of im Marker $_{0}$ eroded by rhombicuboctaedra with radii increasing from 1 to 10 voxels, which is suitable to extract the boundaries of all aluminium particles.

The final image resulting from the merging of these 10 successive watershed transforms is oversegmented. Therefore, this method was combined with a stochastic watershed segmentation, to eliminate the wrong boundaries.

\section{Stochastic segmentation}

The multiscale segmentation leads to an oversegmentation. All the notables boundaries are present, but there is a lot 


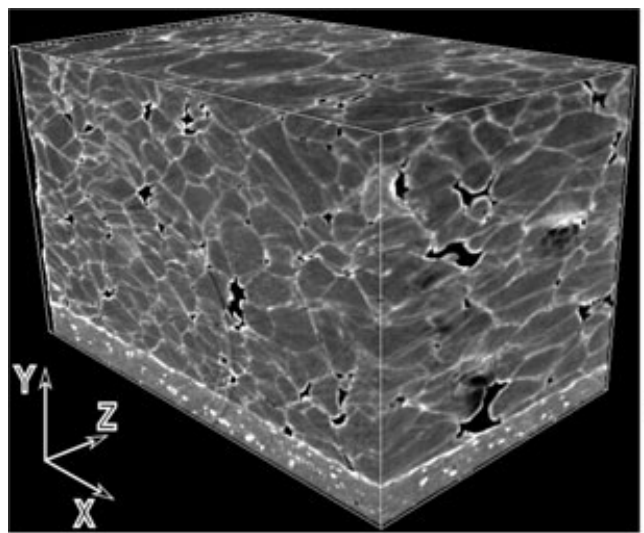

A

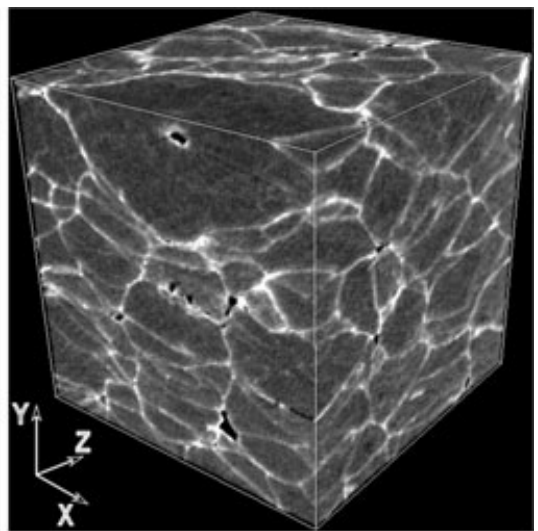

B

Fig. 3. (A) X-ray microtomography image of pure aluminium cold spray coating with particle boundary etching by gallium (Source: ESRF; resolution: $0.35 \mu \mathrm{m} /$ voxel; dimensions: $900 \times 550 \times 551$ voxels $^{3} ; 315 \times 192.5 \times 192.9 \mu \mathrm{m}^{3}$ ); (B) Region of interest into the same image (Source: ESRF; resolution: $0.35 \mu \mathrm{m}$ /voxel; dimensions: $401 \times 401 \times 400$ voxels $^{3} ; 140.4 \times 140.4 \times 140 \mu \mathrm{m}^{3}$ ). Spraying direction along $O y$.

of wrong boundaries. Due to the anisotropy of the material, many of these useless boundaries are planes parallel to the $Y$-axis. For removing these false boundaries, a stochastic watershed is used with anisotropic lines as markers. Namely, lines orthogonal to the $Y$-axis.

The stochastic watershed was proved to be efficient for unsupervised segmentation (Noyel et al., 2007; Faessel \& Jeulin, 2010). The two parameters used for its construction are $k$, the number of random markers used in each realization, and $R$, the number of realizations. From the law of large numbers, the PDF converges when increasing $R$. The parameter $k$ needs to be proportional to the number of desired regions in the segmented image. Therefore, in the case of granular materials, $k$ needs to be proportional to the number of particles contained in the image.
The parameter $k$ can be automatically estimated. In Faessel \& Jeulin (2010), the authors use the covariance for estimating the average radius of the grains, then deduce the number of grains in the image making a Boolean model assumption (Jeulin, 1991). Here, the covariance can't be used: The shape of the grain in the sample is unknown and almost all the voxels are in the same phase (the grains). Instead the granulometry of the initial powder in the cold-sprayed coating is used. Assuming a conservation of the volume of particles during the deposition, the average volume of particles is also conserved, and known from the size distribution of the powder. From the average volume, the number of particles in the region of interest is estimated. This number is the value of parameter $k$ used for the segmentation. The input size distribution of the powder is also used for the validation of the segmentation.

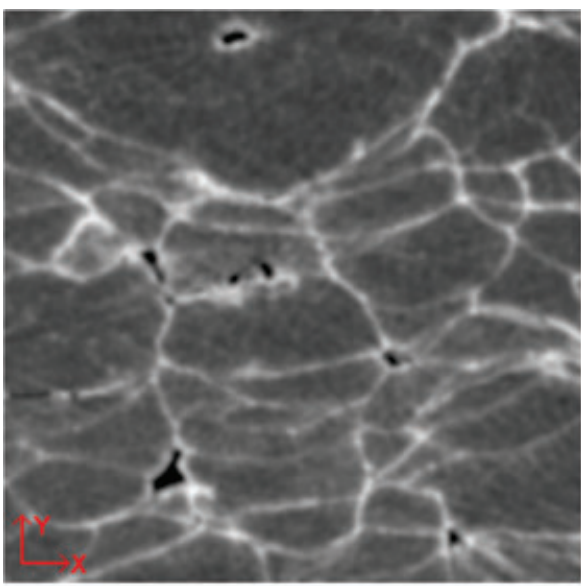

A

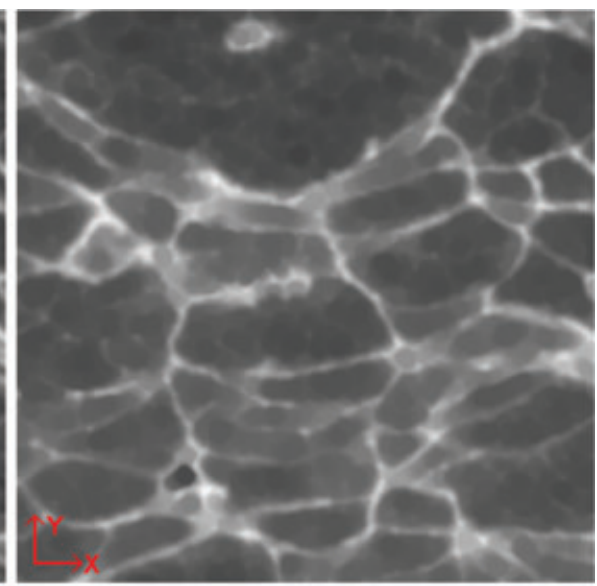

B

Fig. 4. (A) 2D slice of Figure 3(B) in the $x O y$ projection. (B) The same image closed. The structuring element used is a rhombicuboctahedron having a radius equal to 5 voxel $(1.75 \mu \mathrm{m})$. 


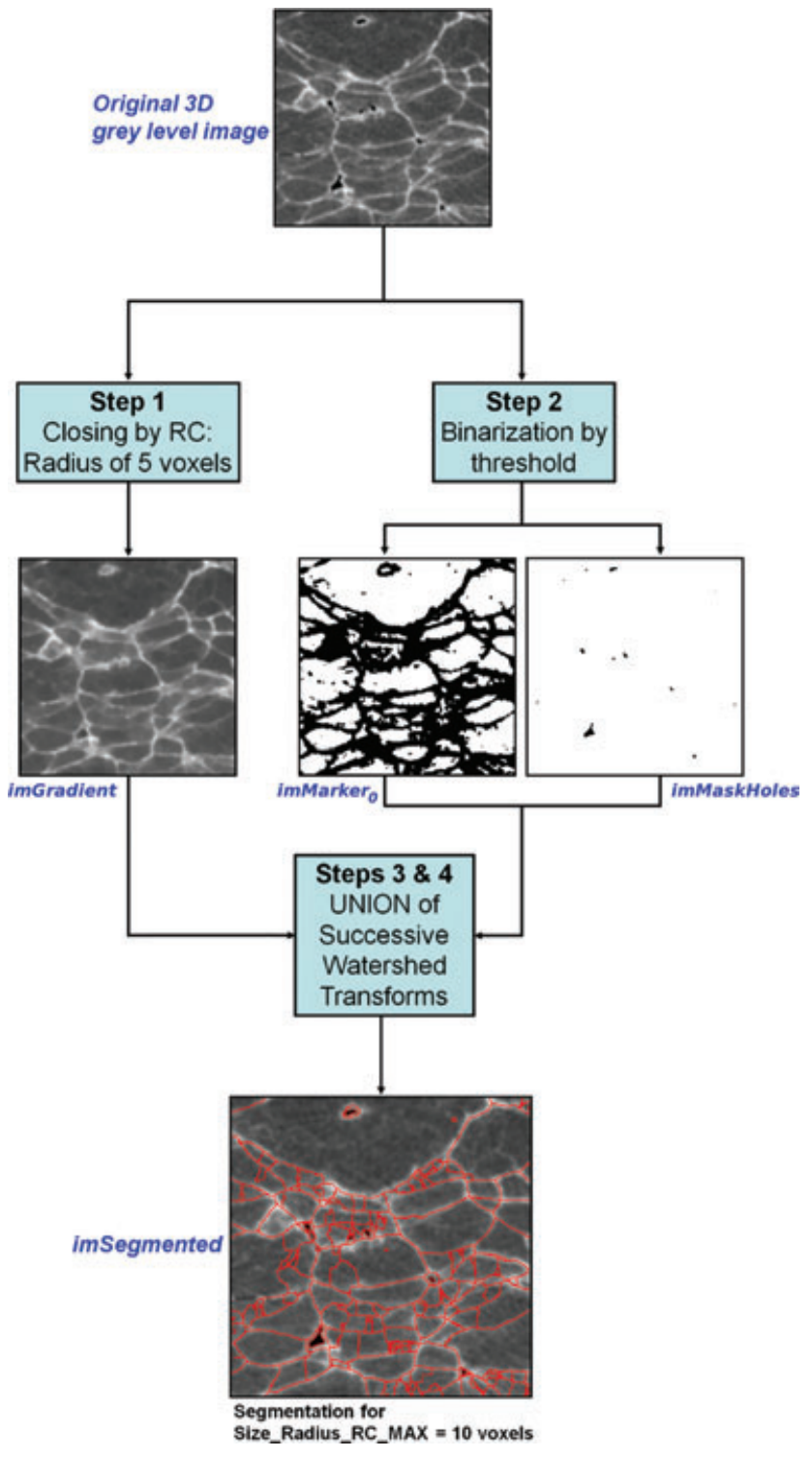

Fig. 5. Multiscale segmentation algorithm.

\section{Stochastic watershed}

The first method introduced for computing the stochastic watershed is based on a large number of realizations of random markers to estimate a PDF of contours, or of surface boundaries in 3D.

In the present case, the markers are random lines parallel to the $X$-axis and lines parallel to the $Z$-axis. These markers will give a high probability of detection of the boundaries parallel to the $X$-axis and the $Z$-axis and a low probability of detection of the boundaries parallel to the $Y$-axis. As illustrated on a 2D simple example, if the markers of a realization are lines parallel to the $X$-axis, the boundaries parallel to the $X$-axis are detected by a constrained watershed, but the the boundaries parallel to the $Y$-axis disappear (Fig. 6).
As in the multiscale segmentation, the topological function used for the watershed construction is a closing of the input image with a small rhombicuboctahedron (radius $=1.75 \mu \mathrm{m}$ ). For each set of markers, a constrained watershed is computed. Then, the Parzen window is used to estimate the PDF of contours (Parzen, 1962).

For a good estimation of the stochastic watershed, 100 to 200 realizations are required (Angulo \& Jeulin, 2007). However, using $\lambda$-flat zones, a stochastic watershed segmentation can be achieved with 50 realizations (Faessel \& Jeulin, 2010). This number is low, but the computation of 50 watersheds is very time consuming, especially on large 3D data sets. Here, 25 realizations with lines parallel to the $X$-axis and 25 realizations with lines parallel to the $Z$-axis are used.

From the PDF, it is possible to obtain the segmentation. The first approach uses this PDF as a topological function for a new watershed (Angulo \& Jeulin, 2007). A more efficient approach uses $\lambda$-flat zones to overcome the fact that the estimated PDF is not constant over each branch of contour (Faessel \& Jeulin, 2010). Illustration of the PDF of contours is give on Figure $7(\mathrm{~A})$, the resulting segmentation is illustrated on Figure 7(B).

\section{Graph-based stochastic watershed}

Direct computation of the probability of a boundary

Computing a large number of watersheds from simulations provides reliable results but is a slow process, especially in $3 D$. A more efficient solution for computing stochastic watersheds is to use a direct approach. Probability of boundaries is directly computed with a good approximation without the use of any realizations (Jeulin, 2008; Stawiaski \& Meyer, 2010).

In the present case, we assume Poisson lines as markers. All the lines are parallel to a given axis denoted $L$. The probability of boundaries can be estimated from the surface area of the opaque projection of the regions on a plane perpendicular to the axis $L$.

The opaque projection for the region $i$ is denoted $P_{L}(i)$ and the surface area is denoted $S\left(P_{L}(i)\right)$.

Given two adjacent regions, the probability $p(i, j)$ of the boundary between regions $i$ and $j$ is obtained from the following equation:

$$
\begin{aligned}
p(i, j)= & \exp \left(-\lambda S\left(P_{L}(i) \cap P_{L}(j)\right)\right)-2 \exp \left(-\lambda S\left(P_{L}(i)\right)\right) \\
& -2 \exp \left(-\lambda S\left(P_{L}(j)\right)\right)+3 \exp \left(-\lambda S\left(P_{L}(i) \cup P_{L}(j)\right)\right. \\
& +\exp \left(\lambda S ( P _ { L } ( i ) \cap P _ { L } ( j ) ) \left[\exp \left(-\lambda S\left(P_{L}(i)\right)\right)\right.\right. \\
& +\exp \left(-\lambda S\left(P_{L}(j)\right)\right) \\
& \left.-2 \exp \left(-\lambda S\left(P_{L}(i) \cup P_{L}(j)\right)\right)\right]
\end{aligned}
$$

where $\lambda$ is the Poisson lines intensity of the considered random lines, and $L$ is the orientation of the lines. When $S\left(P_{L}(i) \cap\right.$ $\left.P_{L}(j)\right)$ can be neglected with respect to $S\left(P_{L}(i)\right)$ and $S\left(P_{L}(j)\right)$, the following approximation of Eq. (1), given in (Jeulin, 2008) 


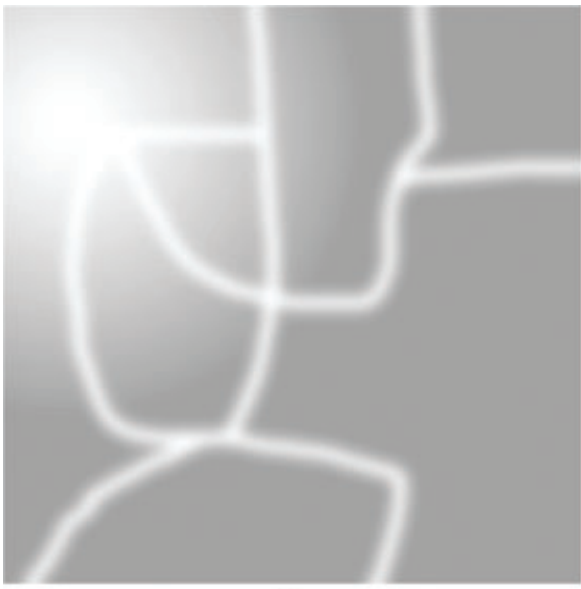

A

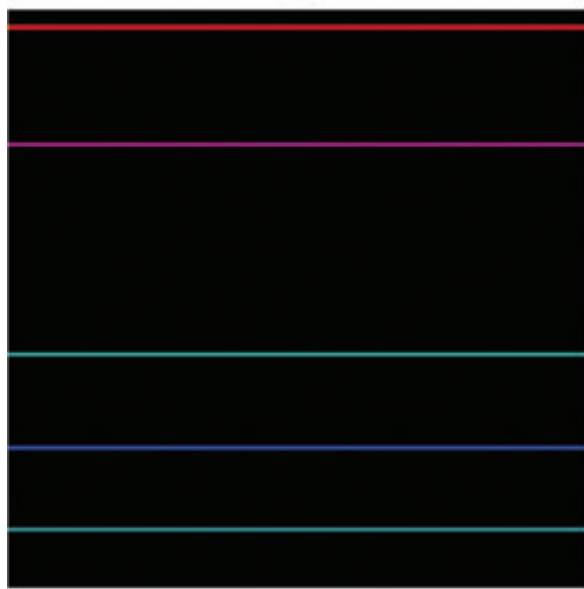

C

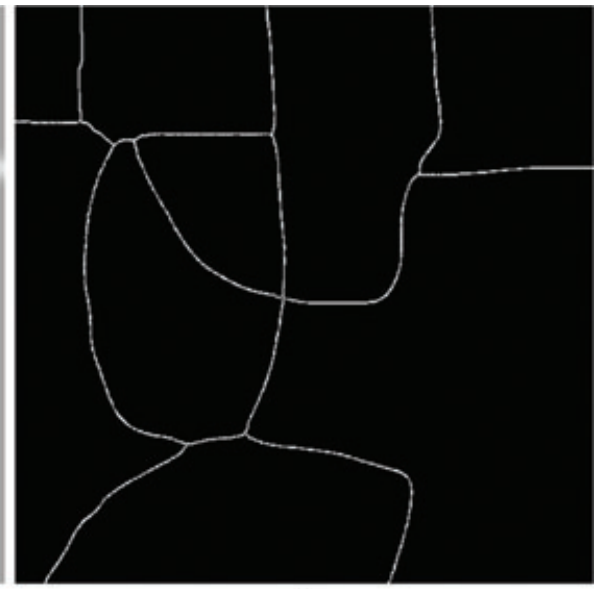

B

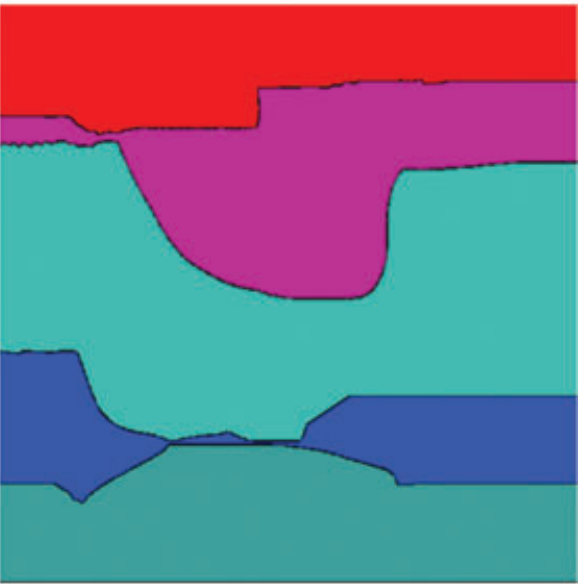

D

Fig. 6. (A) A 2D gradient image. (B) Classical watershed segmentation of the 2D image. (C) The lines parallel to the $X$-axis used as markers of a constrained watershed. (D) Constrained watershed segmentation.

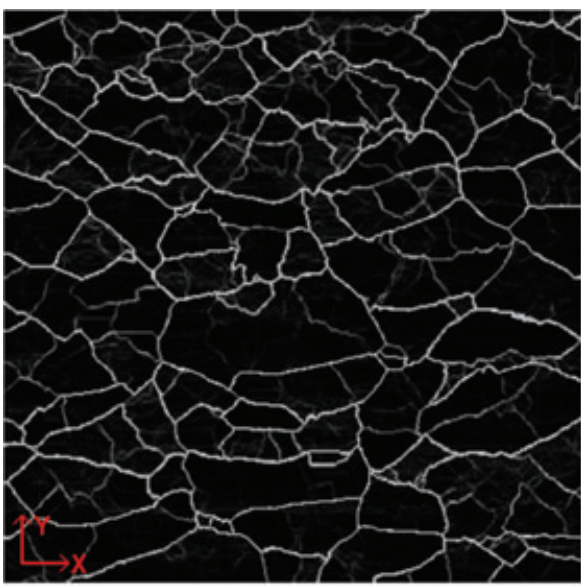

A

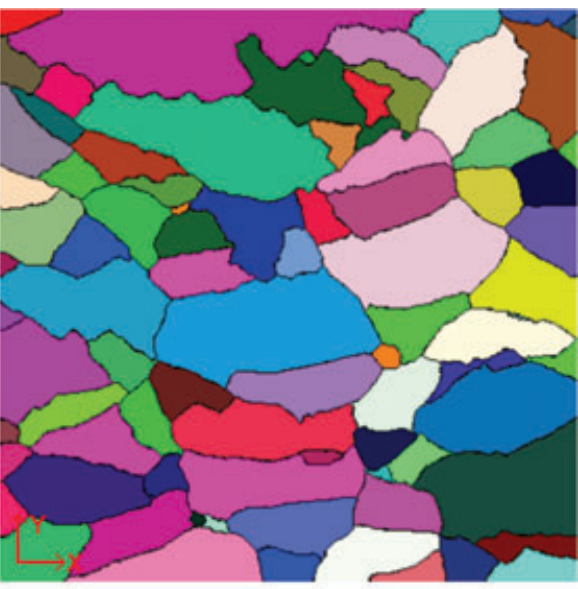

B

Fig. 7. (A) PDF of contours on a X-ray microtomography image of aluminium particles, estimated with 50 realizations of a random process (slice) (B) Stochastic watershed segmentation (slice) The $\lambda$-flat zones are used. 


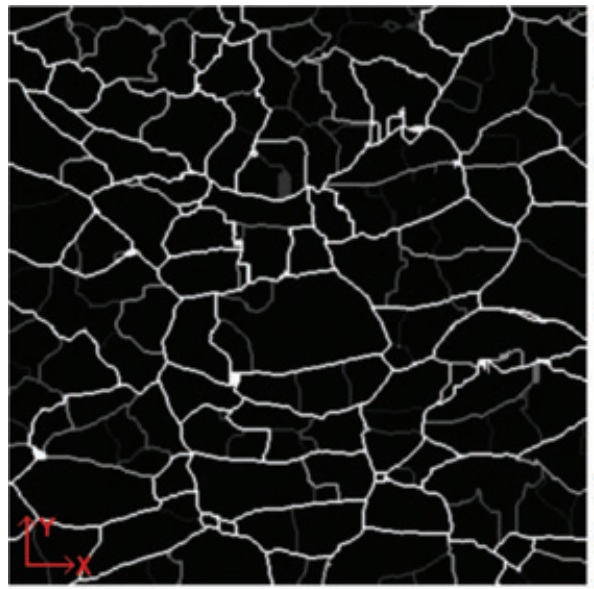

A

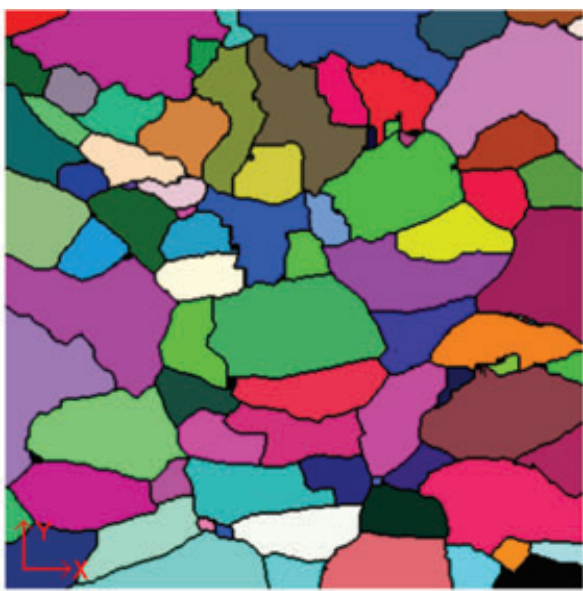

B

Fig. 8. (A) PDF of contours on a X-ray microtomography image of aluminium particles, estimated with the direct, graph-based approach (slice) (B) Stochastic watershed segmentation obtained with a threshold (slice).

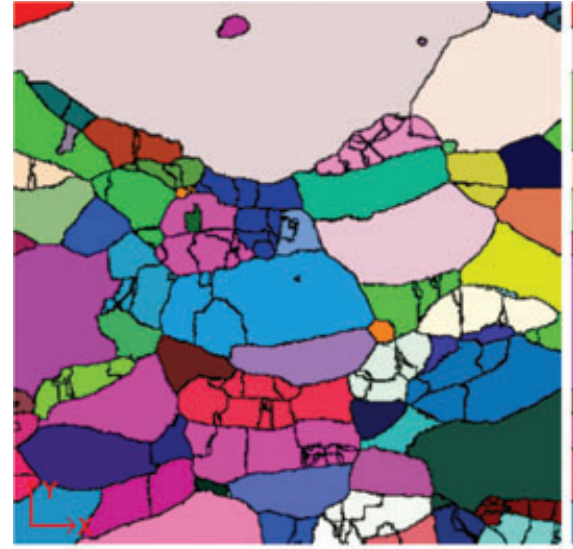

A

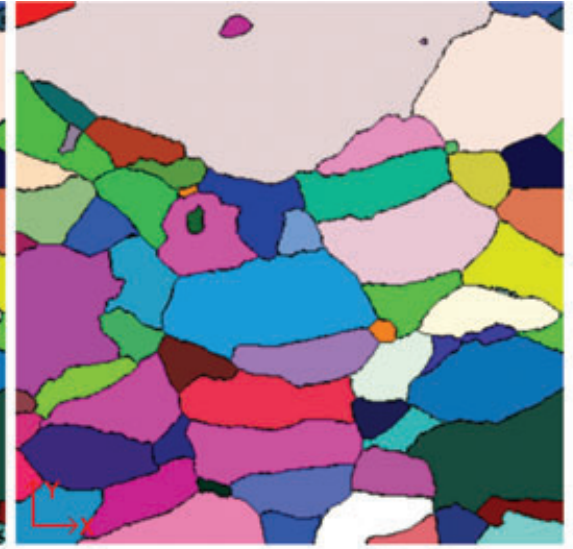

B

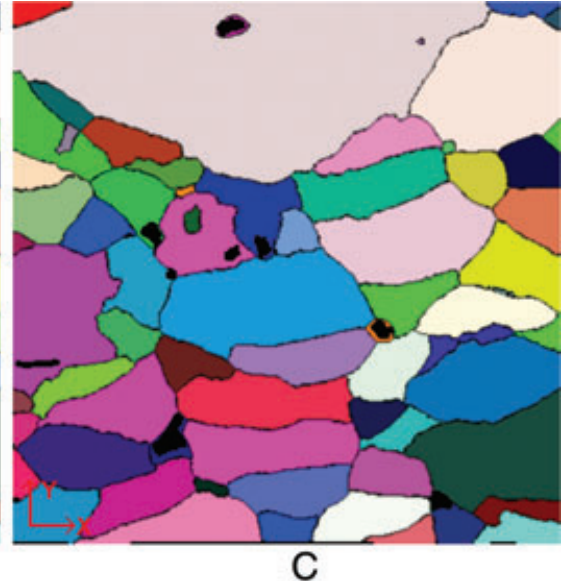

Fig. 9. (A) Combined segmentation before the removal of the internal one-voxel boundaries between merged classes (slice). (B) Combined segmentation before the insertion of the pores (slice). (C) Final combined segmentation.

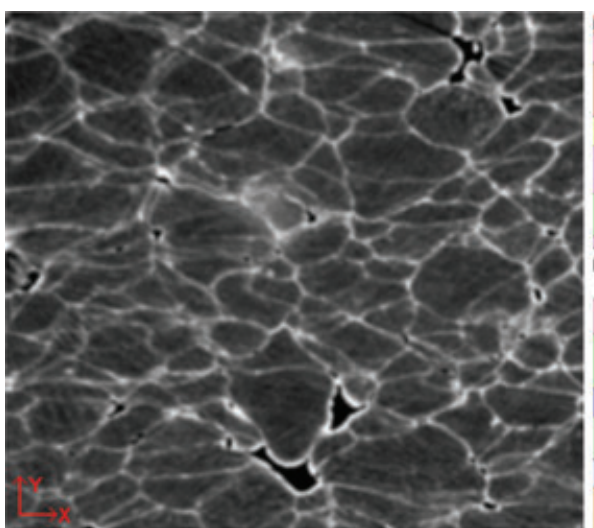

A

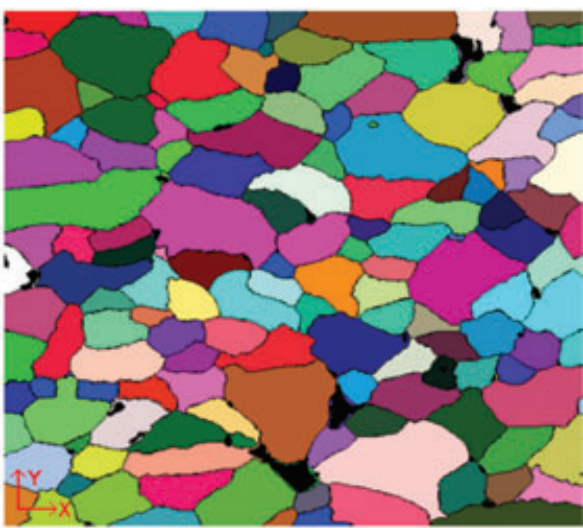

B

Fig. 10. (A) The $185.5 \times 168 \times 99 \mu \mathrm{m}^{3}$ region of interest before segmentation (slice). (B) Final combined segmentation (slice). 
Table 1. Computational cost of the stochastic watershed, the graphbased stochastic watershed, the multiscale image segmentation and the combined segmentation. Times are given for the $140.4 \times 140.4 \times$ $140 \mu \mathrm{m}^{3}$ region of interest on a $3.00 \mathrm{GHz}$ Pentium 4 .

\begin{tabular}{|c|c|}
\hline Algorithm & Time \\
\hline 1 Simple watershed & $3 \mathrm{~min}$ \\
\hline 2 Stochastic watershed & $2 \mathrm{~h} 32 \mathrm{~min}$ \\
\hline 3 Graph-based stochastic watershed & $8 \min 40 s$ \\
\hline 4 Multiscale image segmentation & $31 \mathrm{~min} 40 \mathrm{~s}$ \\
\hline 5 Combination of the partitions & $1 \mathrm{~min} 50 \mathrm{~s}$ \\
\hline 6 Combined segmentation $(3+4+5)$ & $42 \min 10 \mathrm{~s}$ \\
\hline
\end{tabular}

is obtained, andcomment is used in the present segmentation of the cold-spray coating

$$
\begin{aligned}
p(i, j) \simeq & 1-\exp \left(-\lambda S\left(P_{L}(i)\right)\right)-\exp (-\lambda S(P(j))) \\
& +\exp \left(-\lambda S\left(P_{L}(i) \cup P_{L}(j)\right)\right) .
\end{aligned}
$$

\section{Graph-based algorithm}

With the Eq. (2) and a graph-based approach, it is possible to estimate the stochastic watershed by direct computation (Stawiaski \& Meyer, 2010).

As before, the used topological function is a closing of the input image with a small rhombicuboctahedron (radius $=$ $1.75 \mu \mathrm{m}$ ). A first watershed is computed from the local minima of the topological function. Again, a strong oversegmentation is obtained as a result of the presence of noise.

From this watershed, an adjacency graph is constructed. Vertices of the graph are associated to each basin of the watershed, connecting edges between adjacent regions. Values are given to the vertices corresponding to the surface area of the projection of the regions on a plane perpendicular to the axis $L$. The projections $P_{L}(i)$ of all regions $i$ are computed and stored as binary images.
Each edge of the graph is labelled with the minimum of the topological function on the boundary between the corresponding regions. This value corresponds to the height of the gap where the two sources corresponding to the two regions are meeting for the first time during the flooding process of the watershed. From this valued graph, a minimum spanning tree is extracted. Then the regions in the minimum spanning tree are merged, starting with the edge of lowest value.

This merging process corresponds to the natural order of the flooding process in the watershed. The merging process runs until all the nodes of the tree are merged. During this merging process, the probability of the boundaries of the stochastic watershed are estimated and the surface of the projections of the merged regions are updated.

When merging two regions $i$ and $j$, the probability of the corresponding boundary in the stochastic watershed is estimated using the Eq. (2).

The values $S\left(P_{L}(i)\right)$ and $S\left(P_{L}(j)\right)$ are already available. The value $S\left(P_{L}(i) \cap P_{L}(j)\right)$ is deduced from the projections $P_{L}(i)$ and $P_{L}(j)$. The value $S\left(P_{L}(i \cup j)\right)$ is computed, using the values $S\left(P_{L}(i)\right), S\left(P_{L}(j)\right)$ and $S\left(P_{L}(i) \cap P_{L}(j)\right)$, from

$$
\begin{aligned}
S\left(P_{L}(i \cup j)\right) & =S\left(P_{L}(i) \cup P_{L}(i)\right) \\
& =S\left(P_{L}(i)\right)+S\left(P_{L}(j)\right)-S\left(P_{L}(i) \cap P_{L}(j)\right) .
\end{aligned}
$$

Then, the tree and the associated data are updated. The nodes $i$ and $j$ are merged. The new node corresponds to the region $i \cup j$. Therefore, the valuation of this new region is $S\left(P_{L}(i \cup j)\right)$, given by Eq. (3).

After the merging of all the nodes in the original minimum spanning tree, the probability of all the edges of the tree is known. The result is projected from the tree on the graph and from the graph on the image.

Two directions are used tor the random lines, therefore two graph-based stochastic watersheds are computed: one for
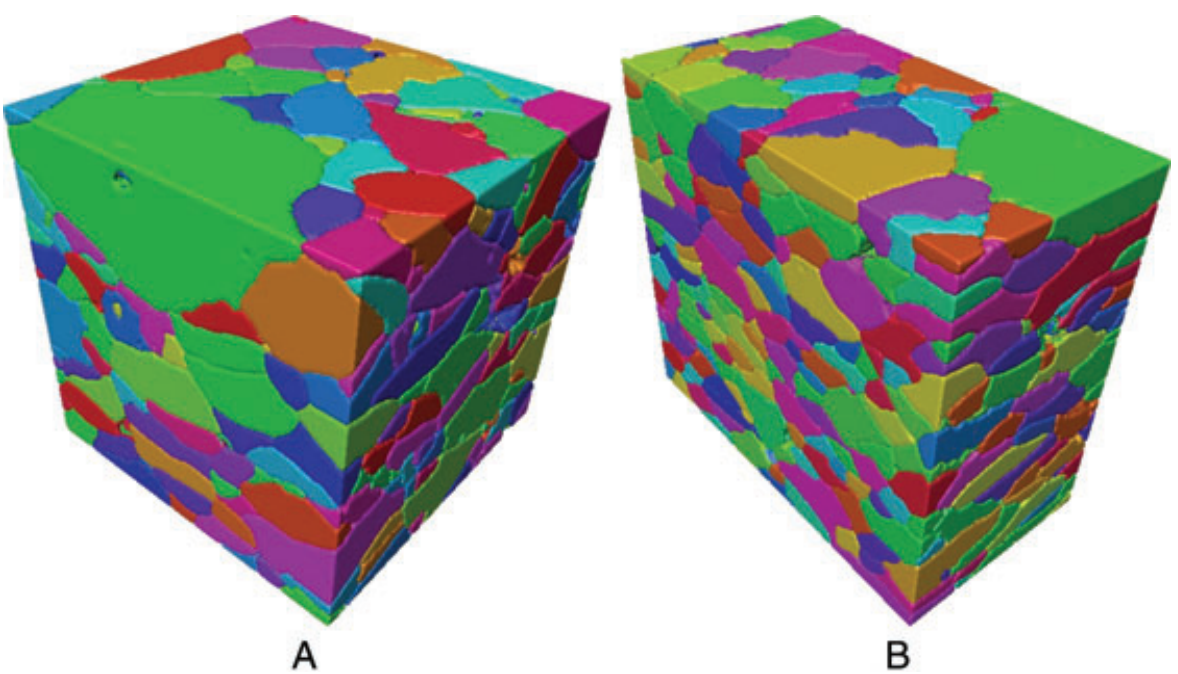

Fig. 11. (A) Combined segmentation for the $140.4 \times 140.4 \times 140 \mu \mathrm{m}^{3}$ region of interest. (B) And for the $185.5 \times 168 \times 99 \mu \mathrm{m}^{3}$ region of interest. Spraying direction along $\mathrm{O} y$. 


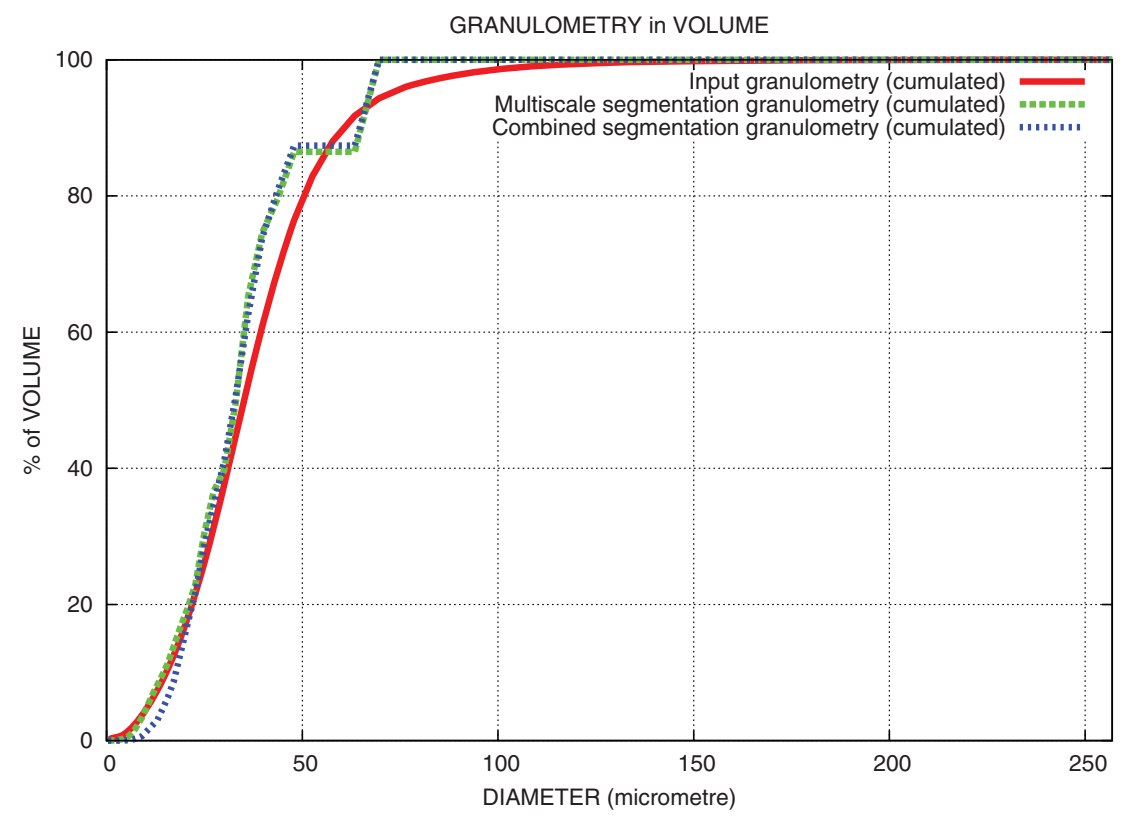

Fig. 12. Cumulative granulometry of the original particles used in the cold-sprayed coating and of the segmented image. Results are given for the multiscale segmentation algorithm and for the combined segmentation algorithm on the $140.4 \times$ $140.4 \times 140 \mu \mathrm{m}^{3}$ region of interest. The MilesLantuéjoul correction is used. each direction. The average of the two PDFs is used as final stochastic watershed.

With a large amount of memory, it is possible to compute single graph-based stochastic watersheds, working with projections in two directions at the same time. During the merging, the probability $p(i, j)$ of the boundary between the corresponding regions $i$ and $j$ is estimated using the following equation:

$$
\begin{aligned}
p(i, j) \simeq & 1-\exp \left[-\lambda\left(S\left(P_{X}(i)\right)+S\left(P_{Z}(i)\right)\right)\right] \\
& -\exp \left[-\lambda\left(S\left(P_{X}(j)\right)+S\left(P_{Z}(j)\right)\right)\right] \\
& +\exp \left[-\lambda\left(S\left(P_{X}(i \cup j)\right)+S\left(P_{Z}(i \cup j)\right)\right)\right] .
\end{aligned}
$$

This approach provides uniform probability on each part of boundary between two regions, as illustrated on Figure 8(A). Therefore, the $\lambda$-flat zones are useless and a simple threshold can be used for the segmentation, as explained later (Fig. 8B). A high threshold gives all boundaries which are not parallel to the $Y$-axis.

\section{Combined segmentation}

We have two segmentations, one stochastic and one multiscale. In both cases, most of the true boundaries are present. In both cases, there is also some oversegmentation.

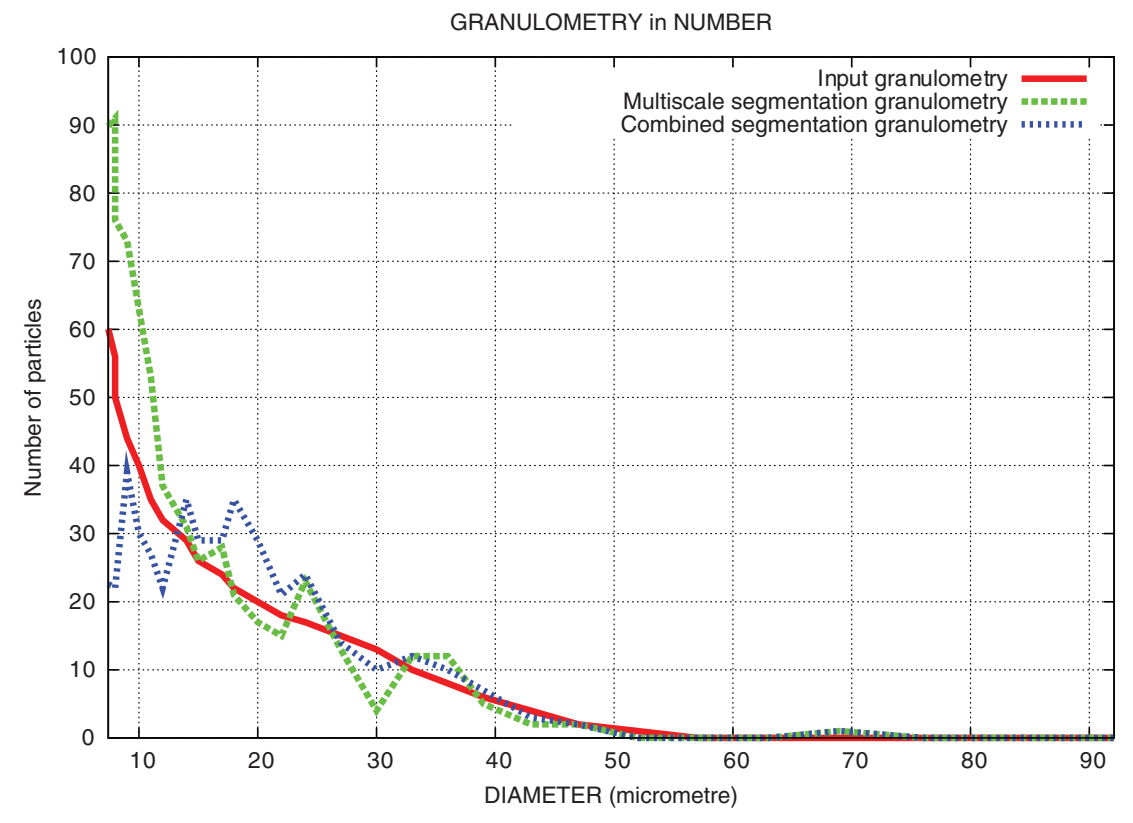

Fig. 13. Granulometry of the original particles used in the cold-sprayed coating and granulometry of the segmented image (given as a histogram of number of particles). Small particles (with a diameter $<7 \mu \mathrm{m}$ ) are ignored. Results are given for the multiscale segmentation algorithm and for the combined segmentation algorithm on the $140.4 \times$ $140.4 \times 140 \mu \mathrm{m}^{3}$ region of interest. 
Fig. 14. Cumulative granulometry of the original particles used in the cold-sprayed coating and of the segmented image. Results are given for the multiscale segmentation algorithm and for the combined segmentation algorithm on the $185.5 \times$ $168 \times 99 \mu \mathrm{m}^{3}$ region of interest. The MilesLantuéjoul correction is used.

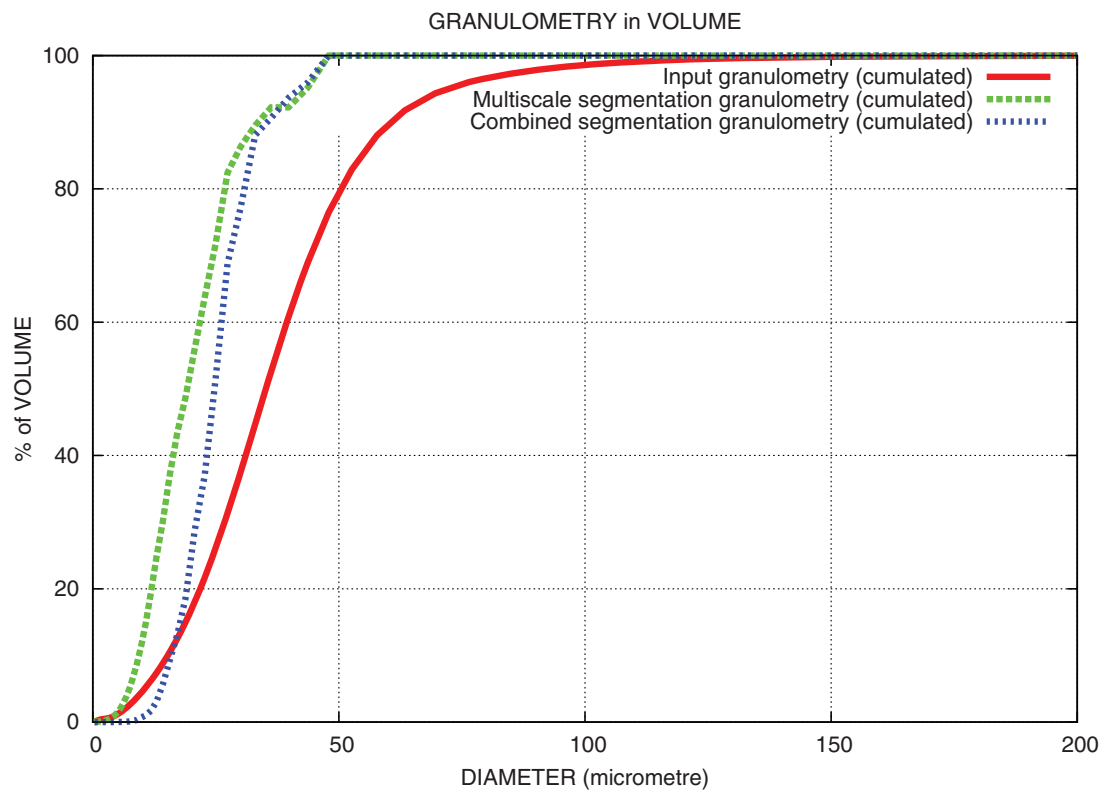

But the false boundaries present in the two segmentations are very different. In the multiscale segmentation, we have mostly some planes parallel to the $Y$-axis. In the stochastic segmentation, we have some very irregular boundaries in the largest particles.

Therefore, it is possible to build a combination of the two segmentations. Boundaries are kept if and only if they are in both segmentations.

Due to some irregularity in the boundaries, it is impossible to achieve such a combination working only on the boundaries. Instead, we work on the partitions, using a supremum. We use the following process: (i) Given two partitions, $P_{1}$ and $P_{2}$, we look for each class of $P_{1}$ its intersection with the classes in $P_{2}$.

(ii) We label each class of $P_{1}$ with the class in $P_{2}$ it predominantly belongs to.

(iii) Then we build the final partition $F$. It is a copy of $P_{1}$, but if two classes in $P_{1}$ predominantly belong to the same class of $P_{2}$, they are merged.

As seen on Figure 9(A), this process is efficient for building the supremum of two partitions, but does not remove the internal one-voxel boundaries between merged classes. For this purpose, we use a last constrained watershed. The markers of this watershed are the merged regions and the topological
Fig. 15. Granulometry of the original particles used in the cold-sprayed coating and granulometry of the segmented image (given as a histogram of number of particles). Small particles (with a diameter $<14 \mu \mathrm{m}$ ) are ignored. Results are given for the multiscale segmentation algorithm and for the combined segmentation algorithm on the $185.5 \times$ $168 \times 99 \mu \mathrm{m}^{3}$ region of interest.

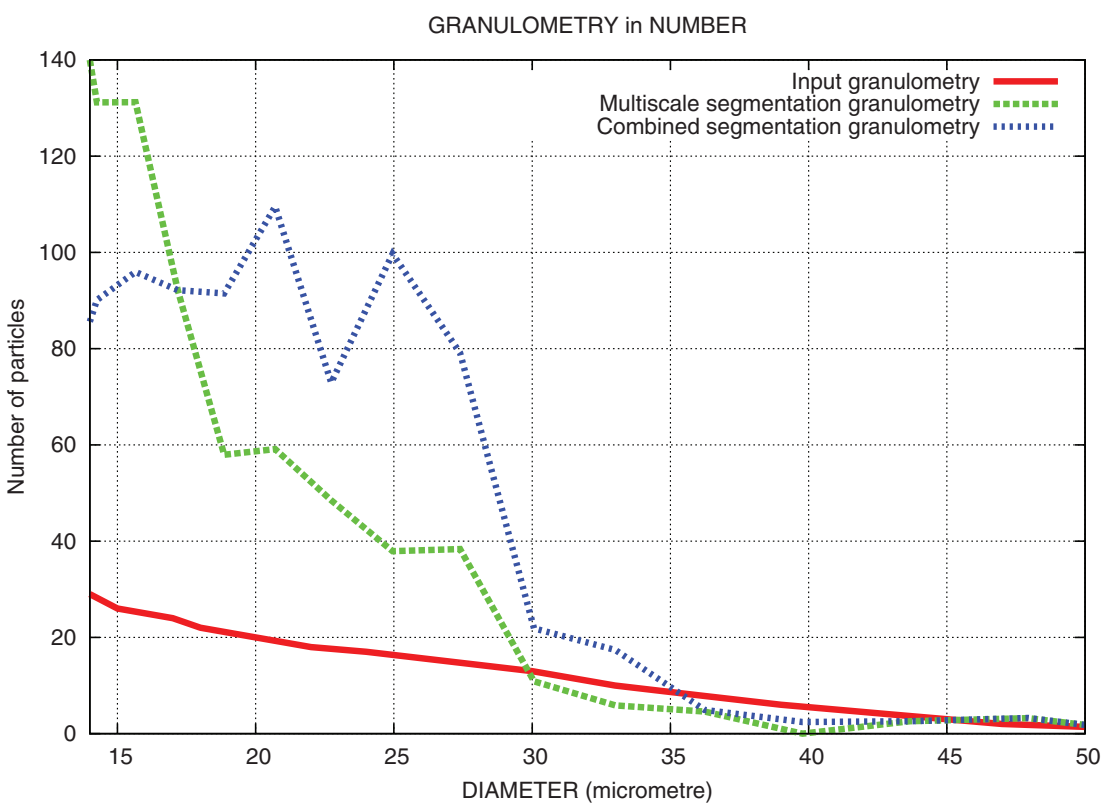




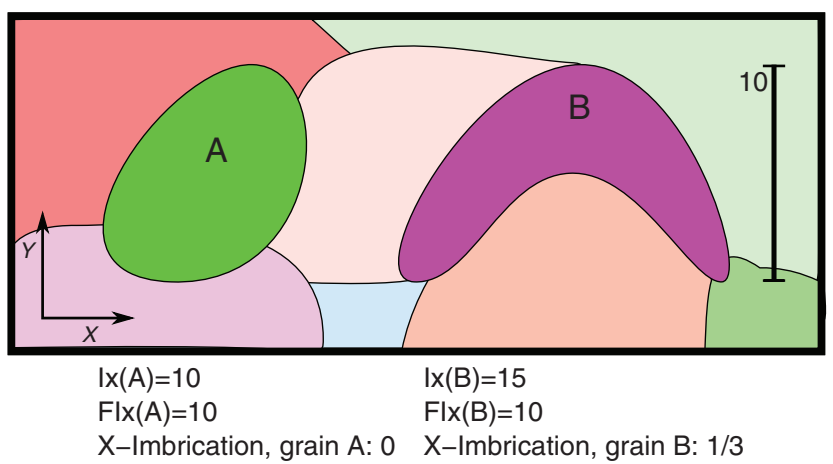

Fig. 16. Illustration of the imbrication measures. The particle $A$ is not imbricated. The particle $B$ has an $X$-imbrication of $1 / 3$. The $Y$ imbrication of both particles $A$ and $B$ is 0 .

function used is the closed input image. The result is on Figure 9(B).

Finally, we build a mask associated to the porosity of the image, using a threshold. With this mask, the pores are inserted in the combination of the two segmentations, leading to the final result. The final segmentation is on Figure 9(C).

Due to the graph approach, the overall process is much faster than a simple stochastic watershed. The combination of the partitions is very fast compared to the others computation, as summarized on Table 1.

\section{Validation on microtomographic images}

We work on the 3D X-ray microtomographic reconstruction of a cold sprayed coating sample presented in section 3.1. We study two regions of interest: the $140.4 \times 140.4 \times$ $140 \mu^{3}{ }^{3}\left(401 \times 401 \times 400\right.$ voxels $\left.^{3}\right)$ region of interest used as illustration (Fig. 3B) and a $185.5 \times 168 \times 99 \mu \mathrm{m}^{3}(530 \times$
$480 \times 283$ voxels $^{3}$ ) region of interest with no large particles (Fig. 10A).

For both regions of interest, we use the same threshold on the PDF of the boundaries in the stochastic watershed segmentation. All the boundaries with a probability higher than $10 \%$ are kept. This threshold is manually selected to insure to keep all significant boundaries. The final segmentation is weakly sensitive to the level of thresholding.

For the multiscale segmentation algorithm, we manually adjust one of the parameters for an optimal segmentation: the number of watershed used in the multiscale process. On the $140.4 \times 140.4 \times 140 \mu \mathrm{m}^{3}$ region of interest, 10 watershed are used. On the $185.5 \times 168 \times 99 \mu \mathrm{m}^{3}$ region of interest, 15 watershed are used. All the others parameters are the same on both regions of interest.

The combined segmentation algorithm provides very good results, as seen on (Figs 9B, 10 and 11). From a 3D visual inspection, along the $X$, the $Y$ and the $Z$ directions, there are some wrong boundaries, and some real boundaries are missing too, but very few of them. On the $140.4 \times 140.4 \times 140 \mu \mathrm{m}^{3}$ region of interest, we find 914 particles. On the $185.5 \times 168 \times$ $99 \mu \mathrm{m}^{3}$ region of interest, we find 1143 particles.

As the granulometry of the original particles used in the cold-sprayed coating is known, it is possible to make a comparison between the granulometry of the segmentations and the original granulometry, assuming spherical shapes for the initial powder and assuming that the volume of the particles is kept after spraying.

As the studied samples are small, we use the MilesLantuéjoul correction to account for the fact that the particles cut by the boundary of the image are deleted from the analysis (Serra, 1982). The idea is to weight each particle $i$ by $P_{i}^{-1}$, $P_{i}$ being the probability that the particle $i$ is not cut by the

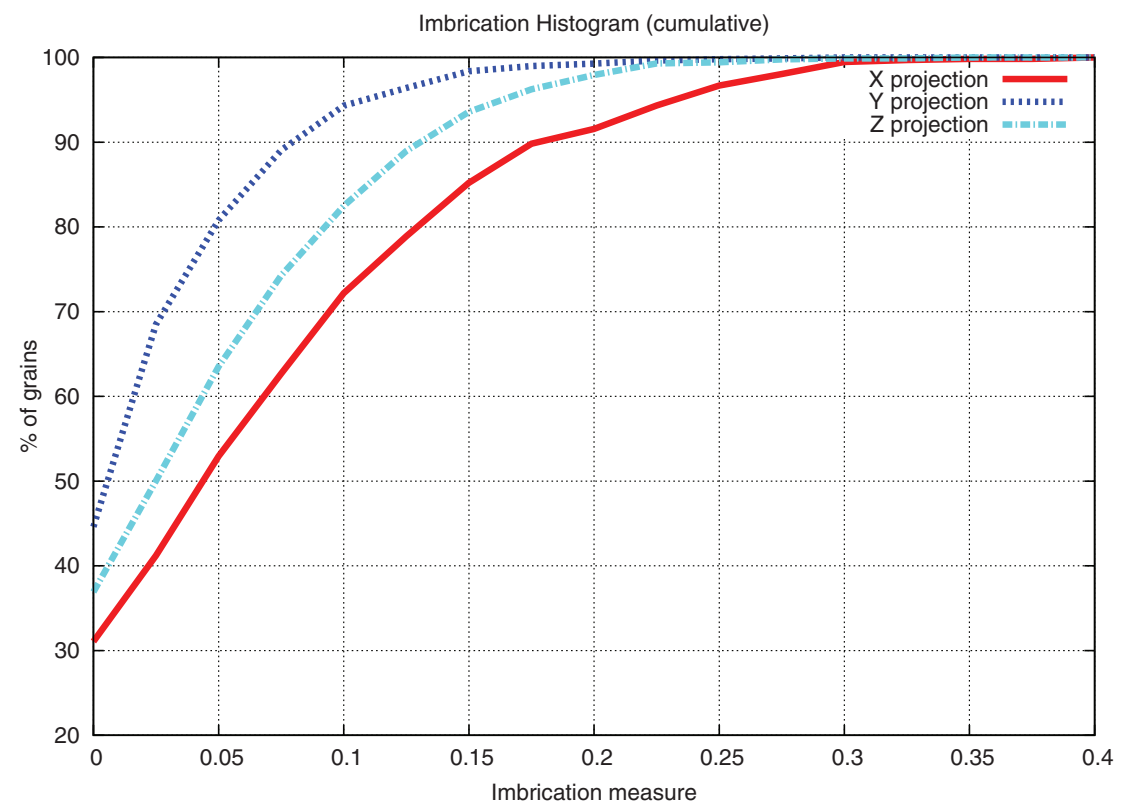

Fig. 17. Cumulative histogram of the measure of the imbrication of the particles. Results are given on the final segmentation of the $140.4 \times 140.4 \times$ $140 \mu \mathrm{m}^{3}$ region of interest. The Miles-Lantuéjoul correction is used. The median $X$-imbrication is 0.049; the median $Y$-imbrication is 0.012 and the median $\mathrm{Z}$-imbrication is 0.026 . 


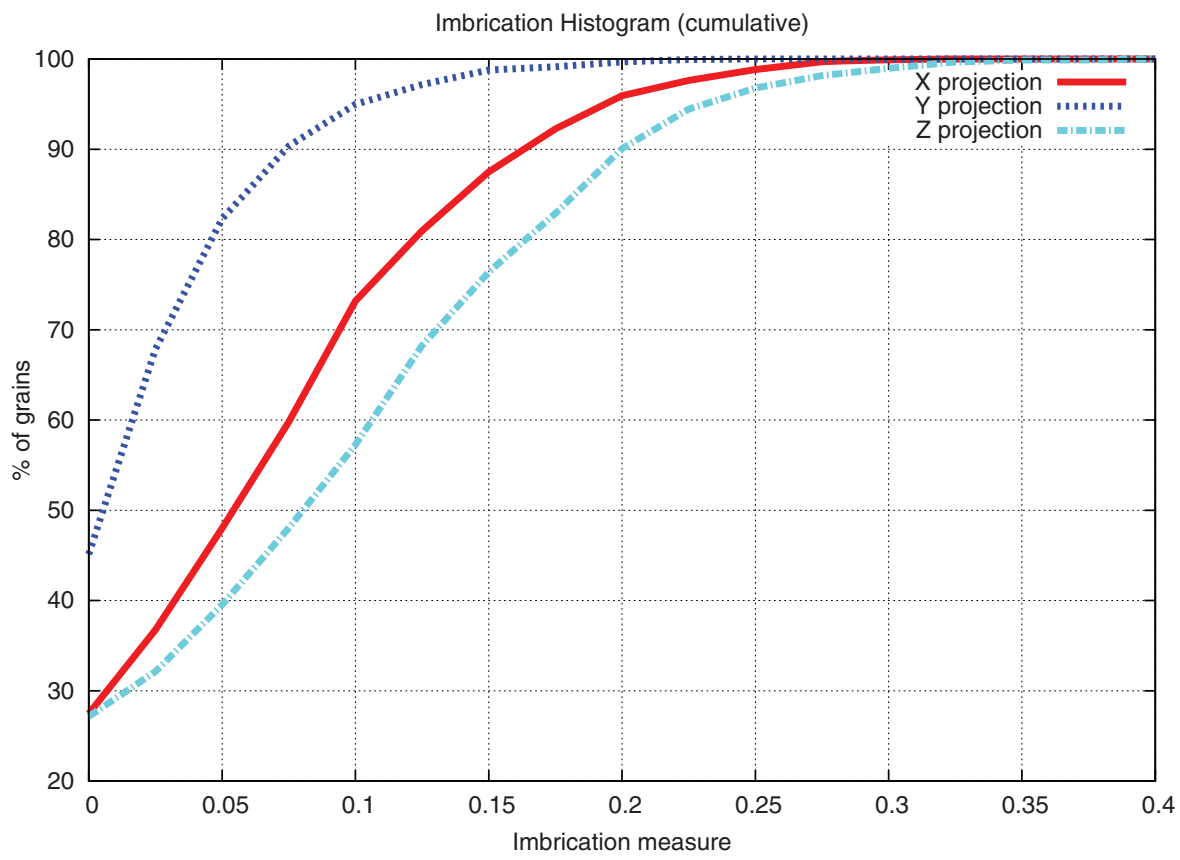

Fig. 18. Cumulative histogram of the measure of the imbrication of the particles. Results are given on the final segmentation of the $185.5 \times 168 \times 99 \mu \mathrm{m}^{3}$ region of interest. The Miles-Lantuéjoul correction is used. The median $X$-imbrication is 0.052 ; the median $Y$-imbrication is 0.012 and the median $\mathrm{Z}$-imbrication is 0.078 .

borders of the sample. The larger the particles are, the smaller $P_{i}$ is.

As seen on Figure 12, the volume granulometry of the powder and of the segmented particles of the coating are in a close agreement for the first region of interest. The granulometry of the multiscale segmentation algorithm is also very close, but the stochastic approach improves the results for medium particles. This improvement can be seen on the number granulometry for particles with a diameter between 7 and $40 \mu \mathrm{m}$ (Fig. 13). The results of the multiscale segmentation algorithm are better for the small particles (smaller than $7 \mu \mathrm{m}$ ).

In the segmentation process, we build a mask associated to the porosity of the image, using a threshold. With this mask, we are able to estimate the volume fraction of the pores. On the $140.4 \times 140.4 \times 140 \mu \mathrm{m}^{3}$ region of interest, the volume fraction of the pores is $1.47 \%$. On the $185.5 \times 168 \times 99 \mu \mathrm{m}^{3}$ region of interest, the volume fraction of the pores is $2.5 \%$.

This result quantitatively shows that the segmentation is correct. Furthermore, the $140.4 \times 140.4 \times 140 \mu \mathrm{m}^{3}$ region of interest is representative of the population of particles used in the cold sprayed coating.

On the $185.5 \times 168 \times 99 \mu \mathrm{m}^{3}$ region of interest, the volume granulometry of the powder and of the segmented particles are not so close (Fig. 14). It does not seem to be a segmentation problem, but instead a sampling problem: A manual check reveals that the second region of interest does not contains any large particle (diameter $>50 \mu \mathrm{m}$ ). The lack of large particles induce a bias in the number of small and medium particles, as highlighted by the granulometry in number (Fig. 15). Using a slice with a thickness limited to 283 voxels is not sufficient in the present case with respect to the largest particles. The volume of the region of interest is sufficient, but its shape is suboptimal. Given a volume, a cubical region of interest provides better results.

\section{Imbrication of the particles}

We want to compute the degree of imbrication of the particles from the microtomographic image of the cold-sprayed coating. For this purpose, new and original imbrication estimators are introduced.

We use the combined segmentation as basis and we study the boundary of each particle. Given a particle $A$ we are able to estimate in 3D the following parameters:

(i) $I x(A)$, the number of entry point, from the left to the right, into the particle $A$ for all the lines parallel to the $X$-axis.

(ii) $F \operatorname{Ix}(A)$, the surface of the $X$-opaque projection of $A$. It is the number of first entry point into the particle $A$ for all the lines parallel to the $X$-axis. We have $F I x(A) \leq I x(A)$.

We define the imbrication of a particle $A$ on the $X$-axis as

$$
1-\frac{F \operatorname{Ix}(A)}{\operatorname{Ix}(A)} .
$$

This measure is similar to the convexity number of the particle, but normalized to 1 and limited to one direction of projection. The imbrication of a convex particle is equal to zero. This 


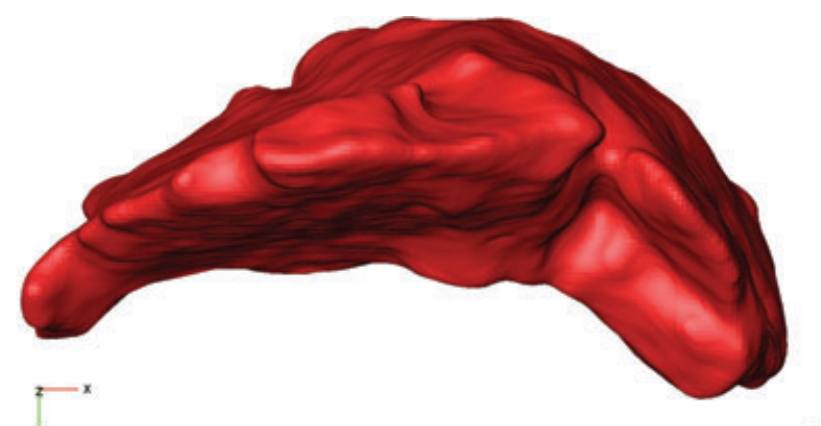

A

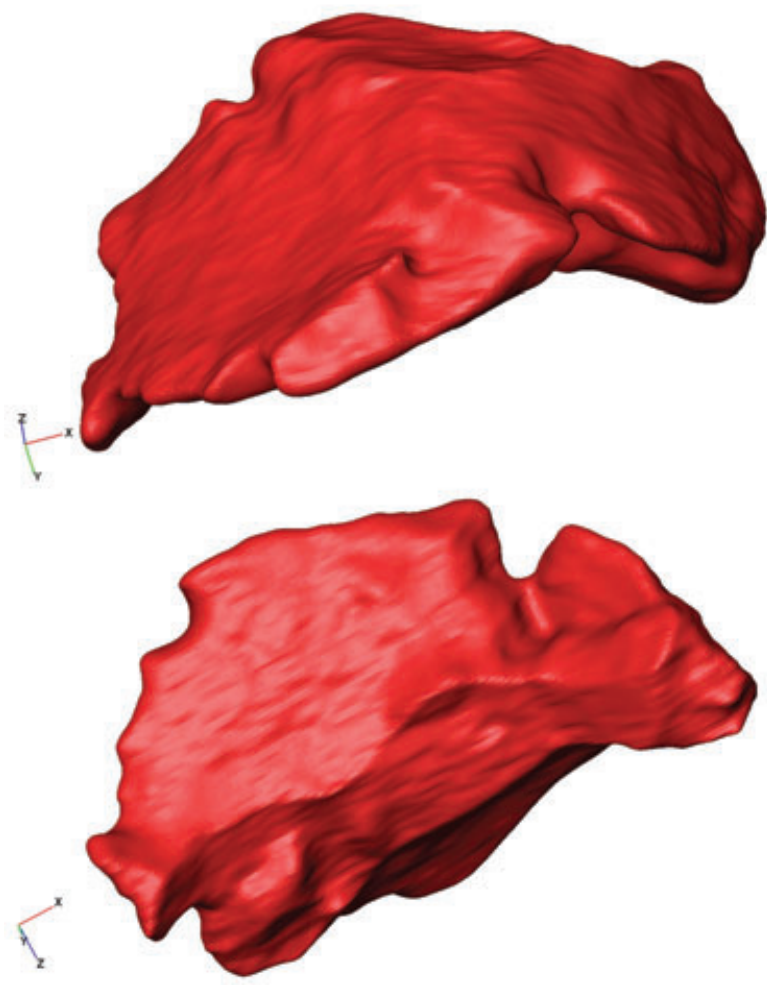

B

Fig. 19. Three different view on a given grain extracted from the final segmentation of the $140.4 \times 140.4 \times 140 \mu \mathrm{m}^{3}$ region of interest. (A) Front view ( $x O y$ projection) (B) Top view. (C) Bottom view. The $X$ imbrication of this grain 0.305 ; the $Y$-imbrication of this grain is 0.031 and the $\mathrm{Z}$-imbrication of this grain is 0.102 .

is illustrated on Figure 16. A real grain extracted from the final segmentation of the $140.4 \times 140.4 \times 140 \mu \mathrm{m}^{3}$ region of interest is illustrated Figure 19.

For each particle, we compute its degree imbrication for the $X$, the $Y$ and the $Z$ projection. The measures on the $140.4 \times 140.4 \times 140 \mu \mathrm{m}^{3}$ region of interest are presented on Figure 17. The measures on the $185.5 \times 168 \times 99 \mu \mathrm{m}^{3}$ region of interest are presented on Figure 18. In both cases, for solving the problem induced by the small sample, we use the Miles-Lantuéjoul correction.

For both regions of interest, the degree of $Y$-imbrication of the particles is less than the degree of $X$ - and $Z$-imbrication, with a median degree of $Y$-imbrication around 0.012 . This is consistent with the cold spray process, as illustrated by the particle $B$ on Figure 16. For both regions of interest, the histograms in $X$ and $Z$ directions are very similar, as a result of the isotropy in the horizontal plane $x \mathrm{Oz}$.

This kind of measurement could be used to compare quantitatively different coatings to give crucial information on the actual anisotropy of the mechanical properties for further optimization of cold spray coatings.

\section{Conclusion}

This new segmentation technique, combining a graph-based stochastic watershed with non-point markers and a multiscale watershed segmentation, is very efficient to extract metallic particles in a cold-sprayed coating.

On the X-ray microtomographic image, the multiscale approach provides a good segmentation. A few boundaries are missing, but it concerns smalls particles. Some large particles are slightly oversegmented.

The granulometry of the segmented image is in a close agreement with the granulometry of the input powder, when the size of the sample is large enough (Figs 12 and 13).

Due to the graph approach, the process is much faster than a simple stochastic watershed, as summarized on Table 1.

Furthermore, a description of the 3D imbrication of metallic particles is proposed, and could help us to predict the strength of the cold-sprayed coating.

\section{Acknowledgements}

Dr. W. Ludwig (MATEIS, ESRF) is warmly thanked for his help for the X-ray acquisition involving Ga penetration. This work was initiated through the 'liaison program' of the Cold Spray Club of Mines ParisTech (http://www.mat.ensmp. fr/clubcoldspray/).

\section{References}

Angulo, J. \& Jeulin, D. (2007) Stochastic watershed segmentation. In Proceedings of the International Symposium on Mathematical Morphology 8 (ISMM) 1, 265-276. Rio de Janerio, Instituto Nacional de Pesquisas Espaciais.

Beucher, S. (1994) Watershed, hierarchical segmentation and waterfall algorithm. Math. Morph. Appl. Image Process. 2, 69-76.

Beucher, S. (2004) Unbiased implementation of the watershed transformation based on hierarchical queues. Tech. rep. CMM/Mines Paristech.

Beucher, S. \& Lantuéjoul, C. (1979) Use of watersheds in contour detection. In Proceedings of the International workshop on image processing, real-time edge and motion detection. Rennes.

Faessel, M. \& Jeulin, D. (2010) Segmentation of 3d microtomographic images of granular materials with the stochastic watershed. J. Microsc. 239, 17-31.

Guipont, V., Jeandin, M., Rolland, G., Jeulin, D., Peyrega, C. \& Ludwig, W. (2010) Microstructures of cold sprayed coatings 
investigated by x-ray microtomography. Ther. Spray Bull. 3, 140147.

Jeulin, D. (1991) Modèles morphologiques de structures aléatoires et de changement d'échelle. Ph.D. Thesis, University of Caen, France.

Jeulin, D. (2008) Remarques sur la segmentation probabiliste. Tech. Rep. N-10/08/MM CMM/Mines Paristech.

Noyel, G., Angulo, J. \& Jeulin, D. (2007) Random germs and stochastic watershed for unsupervised multispectral image segmentation. In Proceedings of Knowledge-Based Intelligent Information and Engineering Systems (KES). Vietri Sul Mare.
Parzen, E. (1962) On estimation of a probability density function and mode. Ann. Math. Stat. 33, 1065-1076.

Rolland, G., Guipont, V., Jeandin, M., Peyrega, C., Jeulin, D. \& Ludwig, W. (2010) Microstructures of cold-sprayed coatings investigated by xray microtomography. In Proceedings of the International Thermal Spray Conference and Exposition (ITSC). Singapore.

Serra, J. (1982) Image Analysis and Mathematical Morphology. Academic Press, London.

Stawiaski, J. \& Meyer, F. (2010) Stochastic watershed on graphs and hierarchical segmentation. In Proceedings ECMI 2010. Wuppertal. 